\title{
Mycotic Spondylitis Caused by Cladosporium Cladosporides: A Case Report
}

\author{
Andrea Burgetova ${ }^{\mathrm{a}, \mathrm{d}}$, Manuela Vaneckova ${ }^{\mathrm{a}}$, Michala Jakubikova ${ }^{\mathrm{b}}$, \\ Zdenek Seidl Prof ${ }^{\mathrm{a}, \mathrm{c}}$
}

\begin{abstract}
Our aim is to show the differences between pyogenic and non-pyogenic spinal inflammation in a magnetic resonance imaging (MRI), which could prompt us toward a diagnosis of the mycotic etiology of the inflammation. We report a patient with the rare mycotic infection Cladosporium Cladosporides, in which one of the manifestations of mycosis is a spinal lesion.Above all, the possible mycotic etiology may be signalled by the relative sparing of the disc, the absence of T2-hyperintense band in the disc and the absence of a post-contrast enhancement of the disc, despite the fact that these symptoms are not specific. We conclude that the mycotic spondylitis is treatable, an accurate substrate diagnosis and adequate clinical guidance shall fundamentally influence the patient's prognosis.
\end{abstract}

Keywords: Fungal infection; Spinal osteomyelitis; Vertebral osteomyelitis; Magnetic resonance imaging; Cladosporium Cladosporides

\section{Introduction}

Mycotic spondylitis is a relatively rare disorder, even if its occurrence has increased with the growing number of patients with an immunological deficit $[1,2]$. We usually contemplate pyogenic etiology of spondylitis or an inflamma-

Manuscript accepted for publication January 30, 2013

\footnotetext{
${ }^{a}$ Department of Radiology, General Teaching Hospital, First Faculty of Medicine, Charles University in Prague, Czech Republic

${ }^{b}$ Department of Neurology, General Teaching Hospital, First Faculty of Medicine, Charles University in Prague, Czech Republic

${ }^{\mathrm{c}}$ Medical College, Prague 5, Czech Republic

${ }^{\mathrm{d}}$ Corresponding author: Andrea Burgetova, MRI Unit, Department of Radiology, General Teaching Hospital and First Faculty of Medicine, Charles University in Prague, Katerinska 30, 12808 Prague 2, Czech Republic. Email: andrea.burgetova@vfn.cz
}

doi: http://dx.doi.org/10.4021/jnr170w tion caused by Mycobacterium tuberculosis, and the correct diagnosis is frequently reached with a considerable delay. Candida and Aspergillus are the most frequently causes of mycotic infections [3, 4]. Szeyko LA et al demonstrated 39 cases of vertebral Coccidiomycosis [5]. We have found no reference in the literature to spondylitis caused by Cladosporium Cladosporides.

\section{Case Report}

A 65-year-old female patient with an infection of the soft facial tissue persisting 8 years and numerous inflammation defects in the calva. The agent of the changes is the mycosis Cladosporium Cladosporoides, which was only recently confirmed by a specialised PCR examination of bone marrow and a sample of tissue from the periphery of the defect in the calva (12/2011). Despite systematic antimycotic therapy (VFEND in a dosage of $400 \mathrm{mg}$ every 12 hours), spondylitis developed (3/2012). The clinical symptoms began with occa-

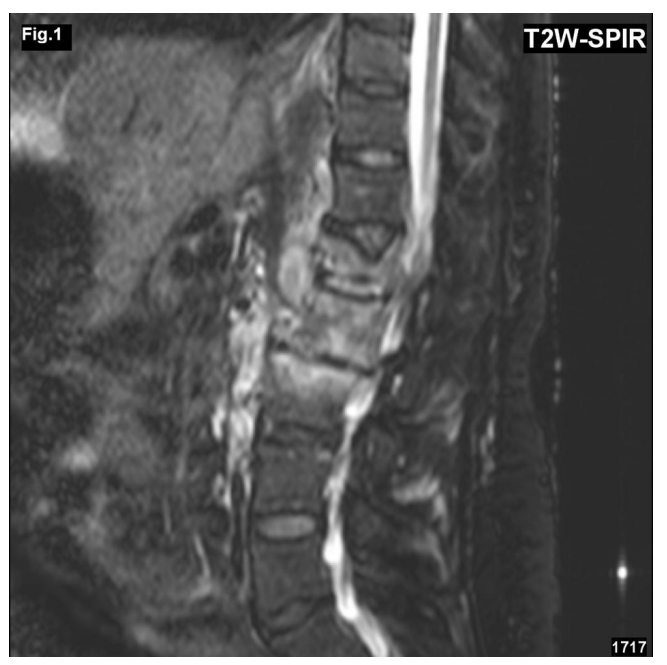

Figure 1. MRI, T2 weighted image with fat suppression (sagittal plane) demonstrated a compression of the vertebral body $L 1$, an increased signal of vertebral bodies L1-3 and in pedicles $L 1$. Intervertebral discs $L 1 / 2$ and $L 2 / 3$ were reduced, hyposignal. 


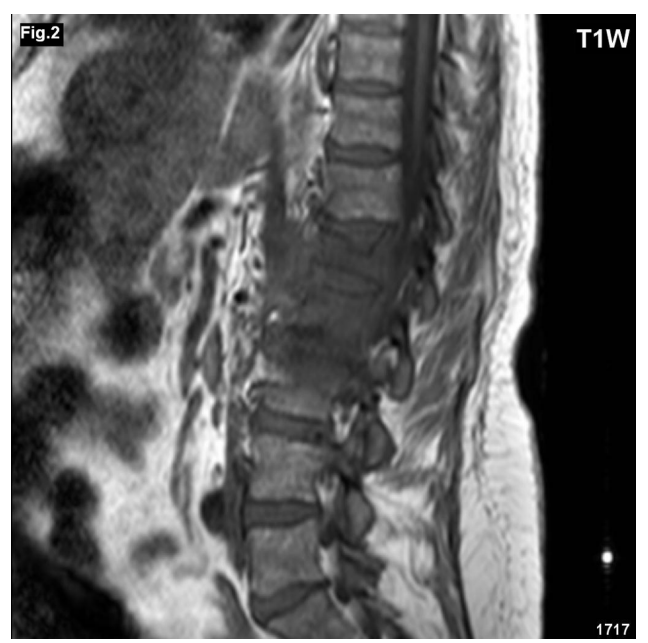

Figure 2. MRI, T1 weighted image (sagittal plane) showed a reduced signal of vertebral bodies L1-3.

sional slight back pain persisting for approximately 2 weeks, gradually there was an accentuation of local pains with partial symptoms of spinal conus (conus medullaris) (weakness in the legs, retention of urine, no defect of sensation). In the laboratory results the inflammation parameters were high (CRP 369.5, leukocytes $\left.13.30 \times 10^{9} / \mathrm{L}\right)$. MRI showed a compression of the vertebral body L1, signal changes of vertebral bodies L1-3 and in pedicles L1. An increased signal in $\mathrm{T} 2$ weighted images (T2WI), more evident in the T2WI with fat suppression (Fig. 1), a reduced signal in T1WI (Fig. 2), after application of contrast agent enhancement of vertebral bodies L1-3, meninges and empyema (Fig. 3a, b). The empyem was most probably largely responsible for the compression of the dural sac and the compression of the spinal conus (on level L1-2). Inflammation penetrated also into the soft paravertebral tissues. Intervertebral discs L1/2 and L2/3 were reduced, hyposignal in the T2WI, after application of contrast agent not enhanced. The patient was indicated for urgent surgical review, but due to the risks of the operational execution a conservative procedure has been chosen.

\section{Discussion}

We do not have any specific non-invasive diagnostic tests or a typical finding in the imaging methods for a diagnosis of mycotic spondylitis. It occurs primarily in patients with an immunological deficit. There is frequently a time lag between clinical symptomatology and diagnosis. The clinical course is frequently subacute, the symptoms are as with vertebrogenic algic syndrome (back pain, radicular irritation syndrome, in rare cases various degrees of neurological deficit [6]. In the imaging methods the finding is generally non-specific, patients are frequently treated empirically with antibiotics for presumed and far more frequent pyogenic infection. In cases where the antibiotic treatment fails and an atypical finding in MRI it is necessary to consider possible mycotic etiology. The diagnosis is achieved by the isolation of the fungus or by the presence of characteristic histopathologic changes, when for confirmation of the diagnosis it is necessary to indicate a percutaneous biopsy under CT control (an open biopsy is less suitable).

In our case we present a patient with a torpid infection persisting for 8 years in the soft facial tissue and the calva, with a known subcutaneous form of the rare mycotic infection Cladosporium Cladosporides. The spinal lesion developed despite continuous systematic antimycotic therapy.
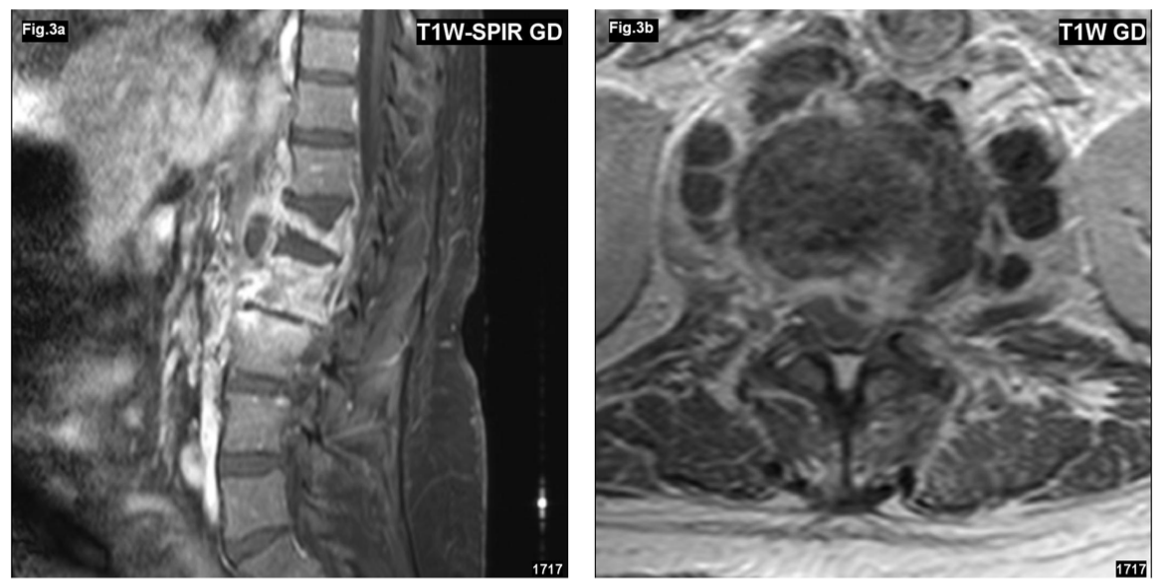

Figure 3. $(a, b) M R I$ after application of contrast agent (sagittal and transversal plane) showed enhancement of vertebral bodies $L 1-L 3$, meninges and empyema. Intervertebral discs $L 1 / 2$ and $L 2 / 3$ were not enhanced. The empyem is responsible for the compression of the dural sac and the spinal conus (on level L1-2). Inflammation penetrated into the soft paravertebral tissues. 
Imaging findings with fungal osteomyelitis can vary from those classically described in association with pyogenic infection. These alterations in appearance may reflect the blunted immune response of the immunocompromised host. In the MRI we wished to demonstrate the possible differences between pyogenic, specific and mycotic spondylitis. In our presented case the lesion was in the vertebrae L1-L3, vertebral body L1 was compressed, there was an evident lesion of pedicle L1, which is rare in pyogenic infections. The affliction of the posterior part of the body and pedicles is more frequently complicated by meningitis, epidural empyema and myelitis [7]. With extensive inflammation inflicting the vertebrae, the discs were relatively well preserved, even if the intervertebral space were considerably reduced. Although the usual finding of low signal intensity in T1WI in discs was present, there was no perceptible post-contrast enhancement. In the T2WI there was a lack of a hyperintense band in the disc. The sign of relative sparing of the disc with absence of a T2-hyperintense band is generally connected with non-pyogenic infection $[1,3]$, but is not unequivocally specific, and has been described also in pyogenic infections [8] and tuberculosis (TBC) [9]. Nevertheless, we believe that the relative sparing of the disc, the absence of a T2-hyperintense band in the disc and the absence of post-contrast enhancement of the disc are radiological signs which should notify us of possible non-pyogenic mycotic etiology of the inflammation. These alterations in appearance may reflect the various immune response of the immunocompromised host [10]. The extensive paraspinal element of the inflammation is also a nonspecific symptom, typically occurring in TBC [11]. In mycotic and bacterial affliction the size of the paraspinal inflammation is mostly dependent on the scope of the bone destruction.

Mycotic spondylitis is treatable, the success of the therapy and the prognosis of the patient depends on timely diagnosis.

\section{Grant Support}

The financial support was from the national research grant agency RVO-VFN64165/2012 and MSMTO21620849.

\section{References}

1. Williams RL, Fukui MB, Meltzer CC, Swarnkar A, Johnson DW, Welch W. Fungal spinal osteomyelitis in the immunocompromised patient: MR findings in three cases. AJNR Am J Neuroradiol. 1999;20(3):381-385.

2. Rossouw I, Goedhals D, van der Merwe J, Stallenberg V, Govender N. A rare, fatal case of invasive spinal aspergillosis in an antiretroviral-naive, HIV-infected man with pre-existing lung colonization. J Med Microbiol. 2011;60(Pt 10):1534-1538.

3. Khazim RM, Debnath UK, Fares Y. Candida albicans osteomyelitis of the spine: progressive clinical and radiological features and surgical management in three cases. Eur Spine J. 2006;15(9):1404-1410.

4. Bertino RE, Porter BA, Stimac GK, Tepper SJ. Imaging spinal osteomyelitis and epidural abscess with short TI inversion recovery (STIR). AJNR Am J Neuroradiol. 1988;9(3):563-564.

5. Szeyko LA, Taljanovic MS, Dzioba RB, Rapiejko JL, Adam RD. Vertebral coccidioidomycosis: presentation and multidisciplinary management. Am J Med. 2012;125(3):304-314.

6. Wrobel CJ, Chappell ET, Taylor W. Clinical presentation, radiological findings, and treatment results of coccidioidomycosis involving the spine: report on 23 cases. J Neurosurg. 2001;95(1 Suppl):33-39.

7. Ehara S, Khurana JS, Kattapuram SV. Pyogenic vertebral osteomyelitis of the posterior elements. Skeletal Radiol. 1989;18(3):175-178.

8. Dagirmanjian A, Schils J, McHenry M, Modic MT. MR imaging of vertebral osteomyelitis revisited. AJR Am J Roentgenol. 1996;167(6):1539-1543.

9. Chapman M, Murray RO, Stoker DJ. Tuberculosis of the bones and joints. Semin Roentgenol. 1979;14(4):266282.

10. DeSanto J, Ross JS. Spine infection/inflammation. Radiol Clin North Am. 2011;49(1):105-127.

11. Whelan MA, Schonfeld S, Post JD, Svigals P, Meisler W, Weingarten K, Kricheff, II. Computed tomography of nontuberculous spinal infection. J Comput Assist Tomogr. 1985;9(2):280-287. 